This is the peer reviewed version of the following article:

Radonjic, A., Vujicic, V., 2020. Integer codes correcting burst asymmetric within a byte and double asymmetric errors. Cryptogr. Commun. 12:221-230.

https://doi.org/10.1007/s12095-019-00388-0

The original version of this article unfortunately contained a mistake in the main title. Instead of "Integer codes correcting burst asymmetric within a byte and double asymmetric errors" the title should read "Integer codes correcting burst asymmetric errors within a byte and double asymmetric errors".

The correction: https://doi.org/10.1007/s12095-019-00398-y

This work is licensed under a Creative Commons Attribution Non Commercial No Derivatives 4.0 license 


\title{
Integer codes correcting burst asymmetric within a byte and double asymmetric errors
}

\author{
Aleksandar Radonjic ${ }^{1}$ Vladimir Vujicic ${ }^{1}$
}

\begin{abstract}
This paper presents a class of integer codes capable of correcting $l$-bit burst asymmetric errors within a $b$-bit byte $(1 \leq l<b)$ and double asymmetric errors within a codeword. The presented codes are constructed with the help of a computer and have the potential to be used in unamplified optical networks. In addition, the paper derives the upper bound on code length and shows that the proposed codes are efficient in terms of redundancy.
\end{abstract}

Keywords Integer codes · Error correction - Asymmetric errors - Optical networks · Theoretical decoding throughput

\section{Introduction}

In most communication networks, it is impossible to predict the error behaviour. More precisely, one cannot know in advance which type of errors will be more frequent $(1 \rightarrow$ 0 or $0 \rightarrow 1$ errors) nor whether they will dominantly affect individual bits or several adjacent bytes.

However, there are networks where error types are known in advance. The bestknown example are unamplified optical networks (UONs) (e.g. local and storage area networks) [1]. In these networks, photons (represented by binary 1's) may fade or fail to be detected, but new photons cannot be generated. So, if the receiver operates correctly, only asymmetric $(1 \rightarrow 0)$ errors may occur $[2,3]$. An additional characteristic of these errors is that they always affect a small number of bits. This was first observed in [4] and then confirmed in [5-9]. In all these studies it was shown that $99.99 \%$ of all errors are either random errors (single or double errors) or bursts of length up to eight bits.

\footnotetext{
Aleksandar Radonjic

sasa_radonjic@yahoo.com

Vladimir Vujicic

vujicicv@yahoo.com
}

1 Institute of Technical Sciences of the Serbian Academy of Sciences and Arts, Belgrade, Serbia 
Aware of this fact, researchers have developed a few classes of codes capable of correcting asymmetric errors [10-13]. Compared to their symmetric counterparts, these codes had simpler structure and lower redundancy. For this reason, they were more suitable for implementation in dedicated hardware. However, it is interesting to mention that none of these codes was ever considered to be used in UONs. The reason for this probably lies in the inability of [10-13] to correct both burst and random asymmetric errors. The mixtures of these errors are not rare, especially in cases when the error rate is high. So, if the codes from [10-13] were used, it would be impossible to provide high throughput and high reliability simultaneously.

Bearing this in mind, in this paper, we present a new class of integer codes called integer $\mathrm{B}_{l / b} \mathrm{AEC}-\mathrm{DAEC}$ codes. The proposed codes, like those in [14-20], have several desirable properties, including the capability to be interleaved without delay. However, unlike [14-20], the proposed codes can correct two types of errors: $l$-bit burst asymmetric errors within a $b$-bit byte $(l / b$ BA errors) $(1 \leq l<b)$ and double asymmetric (DA) errors within a codeword. Owing to this, they are better suited for protection of data in modern UONs.

The organization of this paper is as follows: Section 2 deals with the construction of integer $\mathrm{B}_{l / b} \mathrm{AEC}-\mathrm{DAEC}$ codes. The error correction procedure and theoretical decoding throughputs for these codes are described and evaluated in Section 3, while Section 4 concludes the paper.

\section{Codes construction}

Definition 1 [20] Let $Z_{2^{b}-1}=\left\{0,1, \ldots, 2^{b}-2\right\}$ be the ring of integers modulo $2^{b}-1$ and let $B_{i}=\sum_{n=0}^{b-1} a_{n} \cdot 2^{n}$ be the integer representation of a b-bit byte, where $a_{n} \in\{0,1\}$ and $1 \leq i \leq k$. Then, the code $C(b, k, c)$, defined as

$$
C(b, k, c)=\left\{\left(B_{1}, B_{2}, \ldots, B_{k}, B_{k+1}\right) \in Z_{2^{b}-1}^{k+1}: \sum_{i=1}^{k} C_{i} \cdot B_{i} \equiv B_{k+1}\left(\bmod 2^{b}-1\right)\right\}
$$

is an $(k b+b, k b)$ integer code, where $c=\left(C_{1}, C_{2}, \ldots, C_{k}, 1\right) \in Z_{2^{b}-1}^{k+1}$ is the coefficient vector and $B_{k+1} \in Z_{2^{b}-1}$ is an integer.

The first step to construct integer $\mathrm{B}_{l / b} \mathrm{AEC}-\mathrm{DAEC}$ codes is to determine the integer values of $l / b$ BA and DA errors. When it comes to an $l / b$ BA error, its integer value is already known [19]: it is equal to $e^{\prime}=-2^{r \cdot} \cdot(2 m-1)$, where $0 \leq r \leq b-l, 1 \leq m \leq 2^{v-1}$ and $1 \leq v \leq l$. On the other hand, it is known that a DA error may corrupt one or two $b$-bit bytes. In the first scenario, its integer value will be equal to $e^{\prime \prime}=-2^{s}-2^{r}=-2^{r} \cdot\left(2^{s-r}+\right.$ 1 ), where $0 \leq r<s \leq b-1$. In contrast, if a DA error corrupts two $b$-bit bytes, it will change their integer values by $e^{\prime \prime \prime}=-2^{u}$, where $0 \leq u \leq b-1$. On the basis of this and previous conclusions, we can give the following definitions.

Definition 2 Let $x=\left(B_{1}, B_{2}, \ldots, B_{k}, B_{k+1}\right) \in Z_{2^{b}-1}^{k+1}, y=\left(\underline{B}_{1}, \underline{B}_{2}, \ldots, \underline{B}_{k}, \underline{B}_{k+1}\right) \in Z_{2^{b}-1}^{k+1}$ and $e=y-x=\left(\underline{B}_{1}-B_{1}, \underline{B}_{2}-B_{2}, \ldots, \underline{B}_{k}-B_{k}, \underline{B}_{k+1}-B_{k+1}\right)=\left(e_{1}, e_{2}, \ldots, e_{k}, e_{k+1}\right) \in Z_{2^{b}-1}^{k+1}$ be respectively, the sent codeword, the received codeword and the error vector. Then, an 
$(k b+b, k b)$ integer code is said to be $a\left(B_{l} A E C\right)_{b}-D A E C$ code if it can correct error vectors from the set $E=\left\{\left(e^{\prime}, 0, \ldots, 0,0\right), \ldots,\left(0,0, \ldots, e^{\prime}, 0\right),\left(0,0, \ldots, 0,-e^{\prime}\right),\left(e^{\prime \prime}, 0, \ldots, 0\right.\right.$, $0), \ldots,\left(0,0, \ldots, e^{\prime \prime}, 0\right),\left(0,0, \ldots, 0,-e^{\prime \prime}\right),\left(e^{\prime \prime \prime}, e^{\prime \prime \prime}, \ldots, 0,0\right), \ldots,\left(e^{\prime \prime \prime}, 0, \ldots, e^{\prime \prime \prime}, 0\right),\left(e^{\prime \prime \prime}, 0, \ldots, 0\right.$, $\left.\left.-e^{\prime \prime \prime}\right), \ldots,\left(0, e^{\prime \prime \prime}, \ldots, e^{\prime \prime \prime}, 0\right),\left(0, e^{\prime \prime \prime}, \ldots, 0,-e^{\prime \prime \prime}\right), \ldots,\left(0,0, \ldots, e^{\prime \prime \prime},-e^{\prime \prime \prime}\right)\right\}$, where $e^{\prime} \in\left\{-2^{r}\right.$. $\left.(2 m-1): 0 \leq r \leq b-l, 1 \leq m \leq 2^{v-1}, 1 \leq v \leq l\right\}, e^{\prime \prime} \in\left\{-2^{r \cdot} \cdot\left(2^{s-r}+1\right): 0 \leq r<s \leq b-1\right\}$ and $e^{\prime \prime \prime} \in\left\{-2^{u}: 0 \leq u \leq b-1\right\}$.

Definition 3 The error set corresponding to $l / b$ BA errors is defined as

$$
\varepsilon_{1}=s_{1} \cup s_{2}
$$

where

$$
\begin{gathered}
s_{1}=\left\{-2^{r} \cdot(2 m-1) \cdot C_{i}\left(\bmod 2^{b}-1\right): 0 \leq r \leq b-l, 1 \leq m \leq 2^{v-1}, 1 \leq v \leq l, 1 \leq i \leq k\right\} \\
s_{2}=\left\{2^{r} \cdot(2 m-1)\left(\bmod 2^{b}-1\right): 0 \leq r \leq b-l, 1 \leq m \leq 2^{v-1}, 1 \leq v \leq l\right\}
\end{gathered}
$$

Definition 4 The error set corresponding to DA errors corrupting one b-bit byte $\left(D A_{1}\right.$ errors) is defined as

$$
\varepsilon_{2}=s_{3} \cup s_{4}
$$

where

$$
\begin{gathered}
s_{3}=\left\{-2^{r} \cdot\left(2^{s-r}+1\right) \cdot C_{i}\left(\bmod 2^{b}-1\right): 0 \leq r<s \leq b-1,1 \leq i \leq k\right\} \\
s_{4}=\left\{2^{r} \cdot\left(2^{s-r}+1\right)\left(\bmod 2^{b}-1\right): 0 \leq r<s \leq b-1\right\}
\end{gathered}
$$

Definition 5 The error set corresponding to DA errors corrupting two b-bit bytes $\left(\mathrm{DA}_{2}\right.$ errors) is defined as

$$
\varepsilon_{3}=s_{5} \cup s_{6}
$$

where

$$
\begin{gathered}
s_{5}=\left\{-2^{r} \cdot C_{i}-2^{s} \cdot C_{j}\left(\bmod 2^{b}-1\right): 0 \leq r, s \leq b-1,1 \leq i<j \leq k\right\} \\
s_{6}=\left\{-2^{r} \cdot C_{i}+2^{s}\left(\bmod 2^{b}-1\right): 0 \leq r, s \leq b-1,1 \leq i \leq k\right\}
\end{gathered}
$$

If we compare the sets $\varepsilon_{1}$ and $\varepsilon_{2}$, we will note that they have some common elements. The reason for this is that $\mathrm{DA}_{1}$ errors spaced less than $l$ bits can be treated as $l / b \mathrm{BA}$ errors. Hence, to avoid confusion, we will define the following set of syndromes. 
Definition 6 The set of syndromes corresponding to DA $A_{1}$ errors excluding $l / b$ BA errors is defined as

$$
\varepsilon_{4}=s_{7} \cup s_{8}
$$

where

$$
\begin{gathered}
s_{7}=\left\{-2^{r} \cdot\left(2^{s-r}+1\right) \cdot C_{i}\left(\bmod 2^{b}-1\right): l \leq r+l \leq s \leq b-1,1 \leq i \leq k\right\} \\
s_{8}=\left\{2^{r} \cdot\left(2^{s-r}+1\right)\left(\bmod 2^{b}-1\right): l \leq r+l \leq s \leq b-1\right\}
\end{gathered}
$$

Now we can prove the next theorem.

Theorem 1 The codes defined by (1) can correct l/b BA errors and DA errors iff there exist $k$ mutually different coefficients $C_{i} \in Z_{2^{b}-1} \backslash\{0,1\}$ such that

$$
\begin{aligned}
& \text { 1. }\left|\varepsilon_{1}\right|=\left[2^{l-1} \cdot(b-l+2)-1\right] \cdot(k+1) \\
& \text { 2. }\left|\varepsilon_{3}\right|=\frac{b^{2} \cdot k}{2} \cdot(k+1) \\
& \text { 3. }\left|\varepsilon_{4}\right|=\frac{(b-l+1) \cdot(b-l)}{2} \cdot(k+1) \\
& \text { 4. } \varepsilon_{1} \cap \varepsilon_{3} \cap \varepsilon_{4}=\varnothing
\end{aligned}
$$

where $|A|$ denotes the cardinality of $A$.

Proof The proof for Condition 1 is the same as that given in [19]. Hence, it will be omitted. As far as Condition 2 is concerned, it states that $\mathrm{DA}_{2}$ errors generate $\left(b^{2} \cdot k / 2\right)$ $\cdot(k-1)$ nonzero syndromes. To prove this, observe that the set $\varepsilon_{3}$ can be expressed as

$$
\varepsilon_{3}=\bigcup_{x=1}^{2 k} X_{x}
$$

where

$$
\begin{aligned}
& X_{1}=\left\{-2^{r} \cdot C_{1}-2^{s} \cdot C_{j}\left(\bmod 2^{b}-1\right): 0 \leq r, s \leq b-1,2 \leq j \leq k\right\} \\
& X_{2}=\left\{-2^{r} \cdot C_{1}+2^{s}\left(\bmod 2^{b}-1\right): 0 \leq r, s \leq b-1\right\} \\
& X_{3}=\left\{-2^{r} \cdot C_{2}-2^{s} \cdot C_{j}\left(\bmod 2^{b}-1\right): 0 \leq r, s \leq b-1,3 \leq j \leq k\right\} \\
& X_{4}=\left\{-2^{r} \cdot C_{2}+2^{s}\left(\bmod 2^{b}-1\right): 0 \leq r, s \leq b-1\right\} \\
& \quad \vdots \\
& X_{2 k-3}=\left\{-2^{r} \cdot C_{k-2}-2^{s} \cdot C_{j}\left(\bmod 2^{b}-1\right): 0 \leq r, s \leq b-1, k-1 \leq j \leq k\right\} \\
& X_{2 k-2}=\left\{-2^{r} \cdot C_{k-1}+2^{s}\left(\bmod 2^{b}-1\right): 0 \leq r, s \leq b-1\right\} \\
& X_{2 k-1}=\left\{-2^{r} \cdot C_{k-1}-2^{s} \cdot C_{j}\left(\bmod 2^{b}-1\right): 0 \leq r, s \leq b-1, j=k\right\} \\
& X_{2 k}=\left\{-2^{r} \cdot C_{k}+2^{s}\left(\bmod 2^{b}-1\right): 0 \leq r, s \leq b-1\right\}
\end{aligned}
$$


The elements of the above sets will be nonzero and mutually different only if there exist $k$ coefficients $C_{i} \in Z_{2^{b}-1} \backslash\{0,1\}$ such that

$$
\begin{aligned}
& \bigcap_{x=1}^{2 k} X_{x}=\varnothing \\
& \left|X_{1}\right|=b^{2} \cdot(k-1) \\
& \left|X_{2}\right|=b^{2} \\
& \left|X_{3}\right|=b^{2} \cdot(k-2) \\
& \left|X_{4}\right|=b^{2} \\
& \vdots \\
& \left|X_{2 k-3}\right|=b^{2} \cdot 2 \\
& \left|X_{2 k-2}\right|=b^{2} \\
& \left|X_{2 k-1}\right|=b^{2} \cdot 1 \\
& \left|X_{2 k}\right|=b^{2}
\end{aligned}
$$

As a result, it follows that

$$
\left|\varepsilon_{3}\right|=\sum_{x=1}^{2 k}\left|X_{x}\right|=b^{2} \cdot k+b^{2} \cdot \sum_{n=1}^{k-1} n=\frac{b^{2} \cdot k}{2} \cdot(k+1) .
$$

In a similar way, Condition 3 says that $\mathrm{DA}_{1}$ errors excluding $l / b$ BA errors generate $(b-l+1) \cdot(b-l) \cdot(k+1) / 2$ syndromes that are nonzero. To prove this, note that the set $\varepsilon_{4}$ can be expressed as

$$
\varepsilon_{4}=\bigcup_{y=0}^{2(b-l-1)} Y_{y}
$$

where

$$
\begin{aligned}
& Y_{0}=\left\{-2^{r} \cdot\left(2^{l}+1\right) \cdot C_{i}\left(\bmod 2^{b}-1\right): 0 \leq r \leq b-l-1,1 \leq i \leq k\right\} \\
& Y_{1}=\left\{2^{r} \cdot\left(2^{l}+1\right)\left(\bmod 2^{b}-1\right): 0 \leq r \leq b-l-1\right\} \\
& Y_{2}=\left\{-2^{r} \cdot\left(2^{l+1}+1\right) \cdot C_{i}\left(\bmod 2^{b}-1\right): 0 \leq r \leq b-l-2,1 \leq i \leq k\right\} \\
& Y_{3}=\left\{2^{r} \cdot\left(2^{l+1}+1\right)\left(\bmod 2^{b}-1\right): 0 \leq r \leq b-l-2\right\} \\
& \quad \vdots \\
& Y_{2(b-l-1)-3}=\left\{-2^{r} \cdot\left(2^{b-2}+1\right) \cdot C_{i}\left(\bmod 2^{b}-1\right): 0 \leq r \leq 1,1 \leq i \leq k\right\} \\
& Y_{2(b-l-1)-2}=\left\{2^{r} \cdot\left(2^{b-2}+1\right)\left(\bmod 2^{b}-1\right): 0 \leq r \leq 1\right\} . \\
& Y_{2(b-l-1)-1}=\left\{-2^{r} \cdot\left(2^{b-1}+1\right) \cdot C_{i}\left(\bmod 2^{b}-1\right): r=0,1 \leq i \leq k\right\} \\
& Y_{2(b-l-1)}=\left\{2^{r} \cdot\left(2^{b-1}+1\right)\left(\bmod 2^{b}-1\right): r=0\right\} .
\end{aligned}
$$

Obviously, if the coefficients $C_{i} \in Z_{2^{b}-1} \backslash\{0,1\}$ have values such that

$$
\begin{aligned}
& \bigcap_{y=0}^{2(b-l-1)} Y_{y}=\varnothing \\
& \left|Y_{0}\right|=(b-l) \cdot k \\
& \left|Y_{1}\right|=b-l \\
& \left|Y_{2}\right|=(b-l-1) \cdot k \\
& \left|Y_{3}\right|=b-l-1 \\
& \vdots \\
& \left|Y_{2(b-l-1)-3}\right|=2 \cdot k \\
& \left|Y_{2(b-l-1)-2}\right|=2 \\
& \left|Y_{2(b-l-1)-1}\right|=1 \cdot k \\
& \left|Y_{2(b-l-1)}\right|=1 .
\end{aligned}
$$


then

$$
\left|\varepsilon_{4}\right|=\sum_{y=0}^{2(b-l-1)}\left|Y_{y}\right|=(k+1) \cdot \sum_{y=0}^{b-l-1}(b-l-y)=\frac{(b-l+1) \cdot(b-l)}{2} \cdot(k+1) .
$$

Finally, Condition 4 is a necessary condition for distinguishing $l / b$ BA errors from DA errors. So, $(k b+b, k b)$ integer $\mathrm{B}_{l / b} \mathrm{AEC}-\mathrm{DAEC}$ codes must satisfy all the conditions 1 to 4 . Conversely, the codes satisfying conditions 1 to 4 allow us to distinguish $l / b$ BA errors from DA errors. Then we can correct all $l / b$ BA errors and all DA errors. Therefore, these codes are $(k b+b, k b)$ integer $\mathrm{B}_{l / b} \mathrm{AEC}-\mathrm{DAEC}$ codes.

Theorem 2 Let $\xi=\varepsilon_{1} \cup \varepsilon_{3} \cup \varepsilon_{4}$ be the error set for $\left(k b+b\right.$, kb) integer $B_{l / h} A E C-D A E C$ codes. Then,

$$
\begin{aligned}
|\xi| & =\left|\varepsilon_{1}\right|+\left|\varepsilon_{3}\right|+\left|\varepsilon_{4}\right| \\
& =\left[\frac{2^{l} \cdot(b-l+2)+b^{2} \cdot k+(b-l+1) \cdot(b-l)-2}{2}\right] \cdot(k+1) .
\end{aligned}
$$

Proof This theorem follows directly from Theorem 1.

Now, by knowing the cardinality of $\xi$, we can derive the upper bound on code length.

Theorem 3 For any $(k b+b, k b)$ integer $B_{l / b} A E C-D A E C$ code it holds that

$$
k \leq\left\lfloor\frac{\sqrt{\left(2^{b+1}-z-4\right) \cdot 4 b^{2}+\left(b^{2}+z\right)^{2}}-b^{2}-z}{2 b^{2}}\right\rfloor,
$$

where $z=2^{l} \cdot(b-l+2)+(b-l+1) \cdot(b-l)-2$.

Proof From Definition 1 we know that the total number of nonzero syndromes is $2^{b}-2$. On the other hand, from Theorem 2 we know that the set $\xi$ has $|\xi|=\left(z+b^{2} \cdot k\right) \cdot(k+1) / 2$ nonzero elements. Consequently, we have the inequality

$$
\frac{\left(z+b^{2} \cdot k\right) \cdot(k+1)}{2} \leq 2^{b}-2
$$

from where it follows that

$$
k \leq\left\lfloor\frac{\sqrt{\left(2^{b+1}-z-4\right) \cdot 4 b^{2}+\left(b^{2}+z\right)^{2}}-b^{2}-z}{2 b^{2}}\right\rfloor
$$

From the above it is obvious that the coefficients $C_{i}$ cannot be generated without using a computer. For this reason, it was necessary to perform computer-based searches. For the purpose of this paper, we have restricted ourselves to the codes with parameters $8 \leq$ 
$l \leq 9, b=32$ and $k \leq 32$ (Table 1 ). Besides this, we have partially investigated how the number of $C_{i}$ 's depends on the values of $b$ and $l$. Although the obtained results are far from the theoretical maximum (Table 2 ), the proposed codes are very efficient in terms of redundancy. This can be seen from the fact that two integer $\mathrm{B}_{l / b} \mathrm{AEC}-\mathrm{DAEC}$ codes have $b=2 l$ check bits (the $(32,16)$ integer $\mathrm{B}_{8 / 16} \mathrm{AEC}-\mathrm{DAEC}$ code and the $(36,18)$ integer $\mathrm{B}_{9 / 18} \mathrm{AEC}-\mathrm{DAEC}$ code), while $l$-bit burst error correcting Fire codes use at least $3 l+1$ check bits [21].

\section{Error control procedure and theoretical decoding throughput}

The error correction procedure for the proposed codes is similar to that described in [15]. In short, it consists of two steps: obtaining the error correction data from the syndrome table and executing one of the following operations:

- for $l / b B A$ and $D A_{1}$ errors

$$
B_{i}=\underline{B}_{i}+E_{1}\left(\bmod 2^{b}-1\right), 1 \leq i \leq k+1 ;
$$

$E_{1} \in\left\{2^{r \cdot} \cdot(2 m-1): 0 \leq r \leq b-l, 1 \leq m \leq 2^{v-1}, 1 \leq v \leq l\right\} \cup\left\{2^{r \cdot} \cdot\left(2^{s-r}+1\right): 0 \leq r<s \leq b-1\right\}$

- for $\mathrm{DA}_{2}$ errors

$$
\begin{gathered}
B_{i}=\underline{B}_{i}+E_{1}\left(\bmod 2^{b}-1\right), 1 \leq i \leq k ; \\
B_{j}=\underline{B}_{j}+E_{2}\left(\bmod 2^{b}-1\right), i<j \leq k+1 ; \\
E_{1}, E_{2} \in\left\{2^{u}: 0 \leq u \leq b-1\right\} .
\end{gathered}
$$

To generate the syndome table it is necessary to substitute the values of $l, b$ and $C_{i}$ into (2)-(4) and (8)-(13). In this way, exactly $|\xi|$ relationships (Theorem 2) between the syndrome (element of the set $\xi$ ), error location $(i, j)$ and error vector $\left(E_{1}, E_{2}\right)$ will be

Table 1 First 32 coefficients for some integer $\mathrm{B}_{l / 32}$ AEC-DAEC codes

\begin{tabular}{llllllll}
\hline$l=8$ & & & & & & & \\
515 & 533 & 553 & 603 & 719 & 1153 & 1263 & 1317 \\
4159 & 4747 & 5811 & 7557 & 9121 & 13679 & 18557 & 19741 \\
23951 & 30511 & 31223 & 44615 & 45017 & 49263 & 52075 & 54421 \\
56299 & 69621 & 80371 & 102001 & 105277 & 112425 & 114387 & 144093 \\
$l=9$ & & & & & & & 1483 \\
1027 & 1037 & 1081 & 1167 & 1217 & 1385 & 13233 & 16795 \\
2551 & 3339 & 5295 & 7411 & 10997 & 11365 & 4673 \\
18617 & 26351 & 27609 & 30417 & 39257 & 43611 & 467 & 55825 \\
64389 & 77159 & 89799 & 99699 & 104851 & 107481 & 115583 & 122463 \\
\hline
\end{tabular}


Table 2 Number of coefficients for some integer $\mathrm{B}_{l / b} \mathrm{AEC}-\mathrm{DAEC}$ codes

\begin{tabular}{llllllllllll}
\hline & & $b=16$ & $b=17$ & $b=18$ & $b=19$ & $b=20$ & $b=21$ & $b=22$ & $b=23$ & $b=24$ \\
\hline Theoretical bound & $l=8$ & 17 & 24 & 35 & 48 & 67 & 92 & 126 & 173 & 236 \\
& $l=9$ & 14 & 21 & 31 & 45 & 63 & 89 & 123 & 169 & 233 \\
Computer-search result & $l=8$ & 1 & 2 & 3 & 5 & 7 & 11 & 14 & 17 & 22 \\
& $l=9$ & 0 & 0 & 1 & 3 & 4 & 6 & 9 & 14 & 19 \\
\hline
\end{tabular}

\begin{tabular}{|l|l|l|l|l|}
\hline Elcment of the set $\xi(S)$ & Error location $(i)$ & Error vector $\left(E_{1}\right)$ & Error location $(j)$ & Error vector $\left(E_{2}\right)$ \\
\hline & $-\left\lceil\log _{2}(k+1)\right\rceil \rightarrow$ & & $\left\lceil\log _{2}(k+1)\right\rceil \rightarrow$ \\
\hline
\end{tabular}

Fig. 1 Bit-width of one syndrome table entry

established (Fig. 1). So, when $S \neq 0$, the decoder's task is to find the entry with the first $b$ bits as that of the syndrome $S$. If the syndrome table is sorted (according to the elements of $\xi)$, this task will be completed after $n_{\mathrm{TL}}$ table lookups $\left(1 \leq n_{T L} \leq\left\lfloor\log _{2}|\xi|\right\lrcorner+2\right)[22]$.

To illustrate the effectiveness of this approach, suppose that the network nodes (computers, servers, routers, etc.) are equipped with four-core processors having the same specifications as in [19]. In that case, each node will require one second to decode

$$
G=\frac{\left(3.5 \cdot 10^{9}\right) \cdot 128 \cdot k}{9 \cdot k+29 \cdot n_{T L}+4}
$$

data bits [19]. In addition, in [19] it was shown that each core uses the operation.

$$
S_{t}=\sum_{i=1}^{k} C_{i} \cdot \underline{B}_{4 \cdot(i-1)+t}-\underline{B}_{4 \cdot k+t}\left(\bmod 2^{32}-1\right), t=1,2, \ldots, 4 .
$$

to calculate the syndrome $S_{t}$. Applying these results to the presented theory, we can easily assure that, for smaller values of $k$, all considered codes have the potential to be used in 10G networks (Fig. 2) [1]. In addition, from Fig. 2 we also see that the codes with code rate 1024/1056 have theoretical throughput of $15.91 \mathrm{Gbps}$. Hence, they could be candidates for use in 16G Fibre Channel networks [1]. Finally, from (18) it is evident that all analyzed codes are interleaved at the byte level. Thanks to this, they are able to correct various types of errors, including $t$ DA errors and $t$ BA errors less than $l+1$ bits $(1 \leq t \leq 4)$.

\section{Conclusion}

In this paper, we have presented a new class of integer error control codes. We have shown that the presented codes have three characteristics: first, they can correct $l$-bit burst asymmetric errors within a $b$-bit byte and double asymmetric errors within a codeword, second, they use processor-friendly operations, and third, they can be interleaved without delay and without using dedicated hardware. Owing to these 


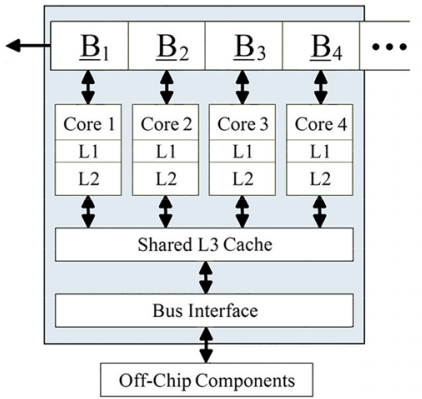

(a)

\begin{tabular}{|c|c|c|c|c|c|}
\hline $\begin{array}{c}\text { Integer } \\
\mathrm{B}_{l / 32} \mathrm{AEC}-\mathrm{DAEC} \\
\text { Code }\end{array}$ & $k$ & $l$ & $\begin{array}{c}\text { Memory } \\
\text { Requirements } \\
\text { for Storing the } \\
\text { Syndrome Table }\end{array}$ & $\begin{array}{c}\text { Number } \\
\text { of Table } \\
\text { Lookups }\end{array}$ & $\begin{array}{c}\text { Minimum } \\
\text { Theoretical } \\
\text { Decoding } \\
\text { Throughput }\end{array}$ \\
\hline$(544,512)$ & 16 & 8 & $2.66 \mathrm{MB}$ & $1 \leq n_{\mathrm{TL}} \leq 19$ & $10.25 \mathrm{Gbps}$ \\
\hline$(544,512)$ & 16 & 9 & $3.35 \mathrm{MB}$ & $1 \leq n_{\mathrm{TL}} \leq 19$ & $10.25 \mathrm{Gbps}$ \\
\hline$(800,768)$ & 24 & 8 & $5.27 \mathrm{MB}$ & $1 \leq n_{\mathrm{TL}} \leq 20$ & $13.44 \mathrm{Gbps}$ \\
\hline$(800,768)$ & 24 & 9 & $6.28 \mathrm{MB}$ & $1 \leq n_{\mathrm{TL}} \leq 20$ & $13.44 \mathrm{Gbps}$ \\
\hline$(1056,1024)$ & 32 & 8 & $8.91 \mathrm{MB}$ & $1 \leq n_{\mathrm{TL}} \leq 21$ & $15.91 \mathrm{Gbps}$ \\
\hline$(1056,1024)$ & 32 & 9 & $10.27 \mathrm{MB}$ & $1 \leq n_{\mathrm{TL}} \leq 21$ & $15.91 \mathrm{Gbps}$ \\
\hline
\end{tabular}

(b)

Fig. 2 a Block diagram of four-core processor and $\mathbf{b}$ theoretical decoding throughputs for some four-byte interleaved integer $\mathrm{B}_{l / 32} \mathrm{AEC}-\mathrm{DAEC}$ codes

features, the presented codes can be transformed into codes capable of correcting various mixtures of asymmetric errors. Such constructed codes could have great potential for practical use, especially in optical networks for short-haul applications.

\section{References}

1. Ramaswani, R., Sivarajan, K., Sasaki, G.: Optical Networks: a Practical Perspective, 3rd edn. Elsevier, Inc., Amsterdam (2010)

2. Pierce, J.R.: Optical channels: practical limits with photon counting. IEEE Trans. Commun. 26, 18191821 (1978)

3. Oprisan, P., Bose, B.: ARQ in optical networks. Proc. IEEE Int'l Symp. Pacific Rim Dependable Computing. 251-257 (2001)

4. Observations of Error Characteristics of Fiber Optic Transmission Systems, CCITT Study Group XVIII Contrib. D21, San Diego, CA, USA (1989)

5. D. Mello, E. Offer and J. Reichert, "Error Arrival Statistics for FEC Design in Four-Wave Mixing Limited Systems," Proc. Opt. Fiber Commun. Conference, pp. 529-530, (2003)

6. L. James, "Error Behaviour in Optical Networks", PhD thesis, Dept. of Engineering, University of Cambridge, (2005)

7. P. Anslow and O. Ishida, "Error Distribution in Optical Links", IEEE 802.3 HSSG Interim Meeting, (2007)

8. Cho, K.Y., Agata, A., Takushima, Y., Chung, Y.C.: Performance of forward-error correction code in 10Gb/s RSOA-based WDM PON. IEEE Photon. Technol. Lett. 22(1), 57-59 (2010)

9. Al-Qazwini, Z., Kim, H.: Ultranarrow Spectrum-sliced incoherent light source for 10-Gb/s WDM PON. J. Lightwave Technol. 30(19), 3157-3163 (2012)

10. Darwish, N., Bose, B.: Efficient double asymmetric error correcting codes. Proc. IEEE Int'l Conf. Computer Design. 166-171 (1989)

11. Y. Saitoh and H. Imai, "Some classes of burst asymmetric or unidirectional error correcting codes," Electron. Lett., vo1. 26, no. 5, pp. 286-287, 1990

12. Bose, B., Al-Bassam, S.: Byte unidirectional error correcting and detecting codes. IEEE Trans. Comput. 41(12), 1601-1606 (1992)

13. Al-Bassam, S., Bose, B.: Asymmetric/unidirectional error correcting and detecting codes. IEEE Trans. Comput. 43(5), 590-597 (1994)

14. Radonjic, A., Vujicic, V.: Integer codes correcting burst errors within a byte. IEEE Trans. Comput. 62(2), 411-415 (2013)

15. Radonjic, A., Bala, K., Vujicic, V.: Integer codes correcting double asymmetric errors. IET Commun. 10(14), 1691-1696 (2016)

16. Radonjic, A., Vujicic, V.: Integer codes correcting spotty byte asymmetric errors. IEEE Commun. Lett. 20(12), 2338-2341 (2016) 
17. Radonjic, A., Vujicic, V.: Integer codes correcting high-density byte asymmetric errors. IEEE Commun. Lett. 21(4), 694-697 (2017)

18. Radonjic, A.: (Perfect) integer codes correcting single errors. IEEE Commun. Lett. 22(1), 17-20 (2018)

19. Radonjic, A., Vujicic, V.: Integer codes correcting burst and random asymmetric errors within a byte. J. Frankl. Inst. 355(2), 981-996 (2018)

20. A. Radonjic and V. Vujicic, "Integer codes correcting sparse byte errors," Cryptogr. Commun., Accepted for publication, https://doi.org/10.1007/s12095-019-0350-9, (2019)

21. Fujiwara, E.: Code Design for Dependable Systems: Theory and Practical Applications. Wiley, Hoboken (2006)

22. Mehlhorn, K., Sanders, P.: Algorithms and Data Structures: the Basic Toolbox. Springer, Berlin (2008) 